S Takci, M Gharibzadeh, M Yurdakok, Z Akcoren, A Korkmaz, S Yigit. Hacettepe University Ihsan Dogramaci Childrens' Hospital, Ankara, Turkey

Objective We aimed to define the etiologic features, outcome of liveborn hydrops fetalis and identify predictors of mortality in a single tertiary unit over an 11-year period.

Method Medical records of liveborn neonates with hydrops fetalis between 2000-2011 were reviewed retrospectively. Demographic data, antenatal interventions clinical manifestations, laboratory findings, outcomes and complete or partial autopsy records were analyzed.

Results Twenty six live born cases of hydrops fetalis with a sex distribution of 33 males and 29 females from the 16,200 live born deliveries in our hospital; an incidence of 3.8/1000 live births. 28 infants $(45 \%)$ were diagnosed as immune hydrops fetalis. Overall, 32 infants (50\%) survived to discharge. The survival rates were comparable within the immune and nonimmune etiology (43\% vs. $53 \%$, $\mathrm{p}>0.05$ ). Of the 34 nonimmune hydrops fetalis (NIHF) cases, a plausible cause could be found for 24 (70.5\%). Post-mortem examinations were performed in 11 cases of 16 non-surviving infants with NIHF. The two factors that were associated independently with mortality in the stepwise multiple logistic regression analyses were a low 5 minute Apgar score and the need for surfactant treatment.

Conclusion Despite the improvements in postnatal care mortality rates are high and treatment options are still limited. The prenatal and postnatal procedures to improve lung capacity and function are necessary to achieve favorable outcome.

\section{VERY LOW BIRTH WEIGHT INFANTS IN ADNAN MENDERES UNIVERSITY NEONATAL INTENSIVE CARE UNIT}

doi:10.1136/archdischild-2012-302724.0801

D Engür, D Çolak, B Çetinkaya Çakmak, S Oktay, M Kaynak Türkmen. Adnan Menderes University, Aydin, Turkey

Background we retrospectively assessed mortality and morbidity rates of very low birth weight infants followed in Adnan Menderes University Neonatal Intensive Care Unit (NICU).

Methods 73 newborns with birth weight lower than $1500 \mathrm{~g}$ and gestational age under 32 weeks followed between January 2009 and December 2011 were included. Demographic characteristics, perinatal events, postnatal complications, duration of mechanical ventilation, mortality rates, length of hospital stay were investigated.

Results Mean gestational age was $28.1 \pm 2.4$ (23-32) weeks, mean birth weight was $1116.8 \pm 247.6(600-1500) \mathrm{g}$, mean mother age was $27.1 \pm 6.2$ (17-41). Median duration of ventilation was $173.4 \pm 182.2$ (0-912) hours. Twenty \% of the cases died; with median gestational age of $26.3 \pm 3.3$ (23-32) weeks and median birth weight of $886 \pm 310$ (600-1450) g. Sixty-four \% of the babies had mechanical ventilation support. Incidence of PDA, NEC, ROP were 19\%, 13.6\%, 19\% respectively. Nineteen \% of the infants had intracranial hemorrhage. Six infants had neurological abnormalities at discharge.

Abstract 801 Table 1 Characteristics of the infants

\begin{tabular}{ll}
\hline & $\%$ \\
\hline male & 55 \\
vaginal delivery & 38 \\
Antenatal Steroid & 30 \\
Mechanical Ventilation & 64 \\
Patent Ductus Arteriosus & 19 \\
Necrotizing Enterocolitis & 13,6 \\
Retinopathy of prematurity & 19 \\
late neonatal sepsis & 23,7 \\
intracranial hemorrhage & 19 \\
\hline
\end{tabular}

Conclusion Overall survival rates of our unit were found to be similar with previously reported rates from our country. Gestational age and birth weight correlated with survival and morbidity rates. Assessing outcomes of our NICU will provide us not only a new approach on understanding and management of these infants but also bring a perspective on predicting the prognosis and informing parents.

\section{CAUSES AND CONTRIBUTING FACTORS LEADING TO EXCHANGE TRANSFUSION :10 YEAR EXPERIENCE AT ADNAN MENDERES UNIVERSITY NEONATAL INTENSIVE CARE UNIT}

doi:10.1136/archdischild-2012-302724.0802

M Kaynak Türkmen, D Engür, D Çolak, S Oktay, A Aydoğdu, A Tosun. Adnan Menderes University, Aydin, Turkey

Background and Aims Jaundice is one of the most common problems in newborn period. Infants at risk for severe hyperbilirubinemia should be identified and closely monitored in order to avoid kernicterus. This study was performed to identify the etiology and sociodemographic characteristics of newborns with severe indirect hyperbilirubinemia who had exchange transfusion.

Methods The study sample included all infants who were undergone exchange transfusion at Adnan Menderes University NICU from January 2000 through December 2010. Hospital records were reviewed. Blood groups, direct Coombs test, whole blood count, peripheric blood smear, CRP, reticulocyte count, total, direct bilirubine levels, Tandem mass, G6PD and pyruvate kinase levels, urinalysis and urine culture, reducing substance in urine were investigated.

Results Sixty-five of the patients were male, 26.5\% preterm. Mean gestational age was $39.3 \pm 1.4$ for term infants, $35 \pm 1.1$ weeks for preterms. Mean serum total bilirubin level was $25 \pm 6.3 \mathrm{mg} / \mathrm{dl}$. Twentyfour \% of the patients had $\mathrm{Rh}$ incompatibility, \%24.5 had $\mathrm{ABO}$ incompatibility, $6.1 \%$ had both $\mathrm{Rh}$ and $\mathrm{ABO}$ incompatibility,\%4.1 G-6-PD deficient, 12.2\% had early breast milk jaundice, $\% 12.2$ had sepsis, $6.1 \%$ had hemolysis of unknown etiology. In five babies no etiology was discovered.

Conclusions Most of the infants had $\mathrm{Rh}$ or $\mathrm{ABO}$ incompatibility, who were vaginally delivered, discharged within 24 hours of birth and exclusively breastfed. Since mean age at admission was $3.4 \pm 3.7$ days, serum total bilirubin levels should be measured before discharge and discharge should be delayed in high risk babies.

\section{EFFECT OF BODY WEIGHT ON THE OUTCOME OF VENTRICULAR SEPTAL DEFECT REPAIR}

doi:10.1136/archdischild-2012-302724.0803

SR Ismail, A Dughiem, R Abusuliman, M Kabbani, H Najm. Cardiac Science, King Abdulaziz Medical City for National Guards, Pediatric Cardiac Intensive Care, Riyadh, Saudi Arabia

Introduction Low body weight and failure to thrive (FTT) often are considered limiting factors for open heart surgery. The purpose of our study is to asses the impact of FTT on ICU outcome of children undergoing Ventricular septal defect repair.

Methods A retrospective Study including all children less than 2 years who had VSD closure by open heart surgery during the period 2002-2010. Children were divided into 2 groups based on their weight for age using standard growth charts. Those with normal or mild failure to thrive ( $Z$ score $>-3$ ) are labeled as group A. group B included all children with severe failure to thrive $(Z$ score $\leq-3)$. Both groups were compared in term of all operative and early postoperative outcome parameters.

Results 145 patients were included. 58 cases in group A and 87 patients in group $\mathrm{B}$. The age and weight in group A was $(8 \pm 5.16$ 